\title{
Téoros
}

Revue de recherche en tourisme

\section{Les Jardins du Québec}

\section{Alexander Reford}

Volume 18, numéro 1, printemps 1999

Les jardins du tourisme

URI : https://id.erudit.org/iderudit/1072295ar

DOI : https://doi.org/10.7202/1072295ar

Aller au sommaire du numéro

Éditeur(s)

Université du Québec à Montréal

ISSN

0712-8657 (imprimé)

1923-2705 (numérique)

Découvrir la revue

Citer cet article

Reford, A. (1999). Les Jardins du Québec. Téoros, 18(1), 5-13.

https://doi.org/10.7202/1072295ar d'utilisation que vous pouvez consulter en ligne.

https://apropos.erudit.org/fr/usagers/politique-dutilisation/ 


\section{$A$ I A I S E}

\section{Les Jardins du Québec}

\section{Alexander Reford}

Les jardins constituent un atout important du paysage du Quebec et un élément de plus en plus indispensable à l'industrie du tourisme. Le Québec est privilégié de posséder une histoire de jardins vieille de plus de quatre siècles, du jardin du Vieux Seminaire à Montréal datant du XVIF siècle aux Grands Jardins de Normandin dont l"aménagement a été complété en 1996. Jusqu'à tout récemment, seuls deux jardins étaient très connus au Québec et à l'extérieur de la province: Le Jardin botanique de Montréal et les Jardins de Métis. Toutefois, le Québéc a été témoin au cours de la dernière décennie de l'aménagement de plusieurs nouveaux jardins et du réaménagement de certains autres. Parallelement, la tenue d'évenements de jardinage de calibre international s'est enracinée dans nos traditions. Aujourd'hui, le Québec jouit d'un réseau de jardins, la plupart des régions de la province en comptent au moins un.

La popularité grandissante du jardinage a eu certains effets sur les jardins du Québec, entre autres, l'accroissement du nombre de visiteurs et de l'attention médiatique. Que le taux d'augmentation du nombre de visiteurs de jardins n'ait pu suivre le rythme de croissance accélérée de l'intérêt pour le jardinage n"est peut-êture pas surprenant. Tant le secteur du marché de gros que le secteur du détail de l'industrie horticole du Québec ont démontré une croissance phénoménale en doublant leurs ventes au cours des dix dernières années! Mais la disjonction apparente entre le jardinage et la visite des jardins dénote une faiblesse dans les efforts de marketing des jardins du Québec pour l'ensemble desquels plusieurs bénéfices de cet intérêt grandissant pour le jardinage, considéré à l'heure actuelle comme l'activité de loisir la plus populaire des Nord-Américains, sont encore à être récoltés.

Encore peu connue à l'extérieur de la province, l'Association des jardins du Québec mobilise ses forces pour se positionner avantageusement sur les marchés touristiques du Canada anglais et des États-Unis. Tourisme Québec lancera très bientôt une importante campagne de promotion ayant pour but de "vendres les du Nord. Les jardins ne sont plus un produit éphémère destiné à une clientèle exclusive. Ils sont devenus un produit en soi et évoluent rapidement comme un des nouveaux choix qui s'offrent aux groupes et aux individus qui visitent le Québec.

\section{LES JARDINS DU QUÉBEC}

Pour les fins de cet article, les jardins comprennent les jardins botaniques et pédagogiques, les jardins historiques et les jardins privés maintenant ouverts au public. L'Association des jardins du Québec compte actuellement vingt membres ${ }^{2}$. Le tableau 1 à la page suivante illustre le nombre de visiteurs payants qu'ont accueillis les jardins membres de l'Association des jardins du Québec. jardins aux jardiniers partout en Amérique
Le Québec compte de nombreux autres jardins dont bon nombre ont été aménagés au cours des cinq dernières années. Certains d"entre eux sont accessibles au public sur' une base régulière et d'autres sur une base limitée. Bien que l'aménagement de tels jardins soit une preuve du rôle de plus en plus important des jardins comme produit touristique, ces jardins n'ont généralement pas été considérés dans cet article 3

\section{LES JARDINS ET LE TOURISME}

Le Québec a été plutôt lent à tirer profit des jardins comme produit touristique. Le Jardin botanique de Montréal a été reconnu comme une mise gagnante importante pour 1a ville, du moins depuis les années 1970. Mais à l'extếrieur de Montréal, les jardins ont généralement été négligés. Durant les années 1970 et 1980 , alors que les jardins zoologiques, les pares et les réserves naturelles prenaient de l'expansion, les musées et les galeries d'art étaient construits et les battiments historiques restaurés, les jardins étaient relégués aux oubliettes. Les jardins appartenant au gouvernement du Québec, tels le domaine Joly-De Lotbinière et le domaine Cataraqui, ont été laissés dans un état presque lamentable. Un grand nombre de jardins historiques, y compris plusieurs situes sur le chemin Saint-Louis à Québec, ont disparu au profit du lotissement et du développement.

Toutefois, depuis la moitié des années 1990, les jardins ont commencé à être développés comme un produit distinct, le Jardin botanique de Montréal en tête avec une série de nouveaux jardins et bâtiments. 


\begin{tabular}{|c|c|c|}
\hline \multicolumn{3}{|c|}{$\begin{array}{c}\text { TABLEAU } 1 \\
\text { Nombre d'entrées aux jardins, } 1998 \text { * }\end{array}$} \\
\hline Arboretum Morgan & Sainte-Anne-de-Bellevue & $6500^{5}$ \\
\hline Domaine Cataraqui & Québec & 44000 \\
\hline Domaine des Fleurs & Saint-Pâcome & 3000 \\
\hline Domaine Joly-De Lotbinière & Sainte-Croix & 18000 \\
\hline Domaine MacKenzie King & Chelsea-Aylmer & 60000 \\
\hline Domaine Maizerets & Québec & 200000 \\
\hline Jardin botanique de Montréal & Montréal & $1007000^{\circ}$ \\
\hline Jardin Van den Hende & Université Laval & 35000 \\
\hline Jardins à Fleur d'eau & Stanbridge East & 4000 \\
\hline Jardins de Métis & Grand-Métis & 102000 \\
\hline Grands Jardins de Normandin & Normandin & 33000 \\
\hline Maison Henry Stuart & Québec & 2000 \\
\hline Parc Marie-Victorin & Kingsey Falls & 24000 \\
\hline La Roseraie de Témiscouata & Cabano & 9000 \\
\hline Seigneurie des Aulnaies & Saint-Roch-des-Aulnaies & $16000^{7}$ \\
\hline Villa Bagatelle & Québec & 3300 \\
\hline Total & & 1566800 \\
\hline
\end{tabular}

exceptionnelles, ces jardins font face à un important problème de promotion. Pour les touristes américains qui peuvent admirer dans la plupart des villes américaines des jardins botaniques, des arboretums et même des aménagements paysagers d'Olmsted, aucun jardin de Montréal ne se distingue comme étant unique. En dépit de leur richesse historique, les jardins de l'île de Montréal n'ont pas toujours été bien traités. II reste quelques jardins historiques, mais aucun n'est accessible au public. En ce sens, Montréal pourrait développer un jardin historique au Manoir MacDougall à Saraguay, propriété de la Communauté urbaine de Montréal.

De plus, très peu de jardins réussissent à captiver les gens intếressés à la conception et à l'architecture du paysage. Peu de projets de développement public et privé à Montréal ont été jumelés à des projets d'aménagement paysager d'intérêt ou d'envergure internationale. À cet égard, le Centre canadien d'architecture, plus intéressant pour les connaisseurs que pour le jardinier moyen, est une exception notable. Depuis 1995, le seul article traitant d'un jardin québécois à paraître dans le Landscape Architecture, la publication de l'American Society of Landscape Architects, est wsweet Farm* dans les Cantons de l'Est ${ }^{10}$. Ce magazine traite régulièrement des jardins urbains et des parcs publics. En améliorant la qualité de ses jardins urbains et en réaménageant ses et ils ont tó finanés par la Ville de Montréal ( 10,7 millions $\$$ ), les gouvernements provincial et fédéral et des sources privées.

\section{LA PROMOTION DES JARDINS DE MONTRÉAL}

En 1997, l'Office des Congrès et du Tourisme du Grand Montréal (OCTGM) a entrepris une étude dans le cadre de ses recherches en "micro-marketing* de sa base de données de clients ${ }^{8}$. Cinq marchés spécifiques ont été identifiés comme ếtant particulièrement prometteurs : wle tourisme gai , la gastronomie, les garderner's clubs, le tourisme culturel et les parieurss" . Les gardener's clubs ont été reconnus comme étant une clientèle susceptible de choisir Montréal pour des séjours de 3 à 4 jours (États-Unis) et jusqu'à 10 jours (Europe). Les efforts de promotion devaient atteindre le nombre élevé d'Américains membres de clubs de jardins, d'associations

horticoles ou abonnés à l'une des nombreuses publications spécialisées en jardinage. C'est sur le Jardin botanique que l'on misait pour inciter les visiteurs à choisir Montréal comme destination pour un séjour de plusieurs jours. Comme initiative visant à promouvoir Montréal, I'OCTGM a fait paraître de la publicité dans des publications américaines de jardinage haut de gamme, comme le Garden Design (mai 1998). Dans sa publicité, Montréal s"affichait comme kla ville des festivals plutôt que comme une ville de jardins. On restait muet sur le Jardin botanique ou sur tout autre attraction touris= tique.

La faiblesse du produit de jardin sur l'île de Montréal explique sans doute ce mutisme. Mis à part le Jardin botanique, il y a peu de jardins intéressants ouverts au grand public. Les jardins ou les aménagements paysagers intéressants sont soit inconnus des touristes, soit dans un état nécessitant des travaux de réaménagement. L'Arboretum Morgan à Sainte-Anne-de-Bellevue et le parc du Mont-Royal ne sont pas connus des touristes. Bien que l'Arboretum Morgan soit le plus grand en son genre au Canada, il demeure plus populaire auprès des résidants que des touristes. Le pare du Mont-Royal a été conçu par Frederick Law Olmsted, pionnier de l'architecture paysagiste, dont le travail est de plus en plus reconnu et apprécié, mais les publications touristiques de la ville passent ce pare sous silence. En dépit de leurs qualités

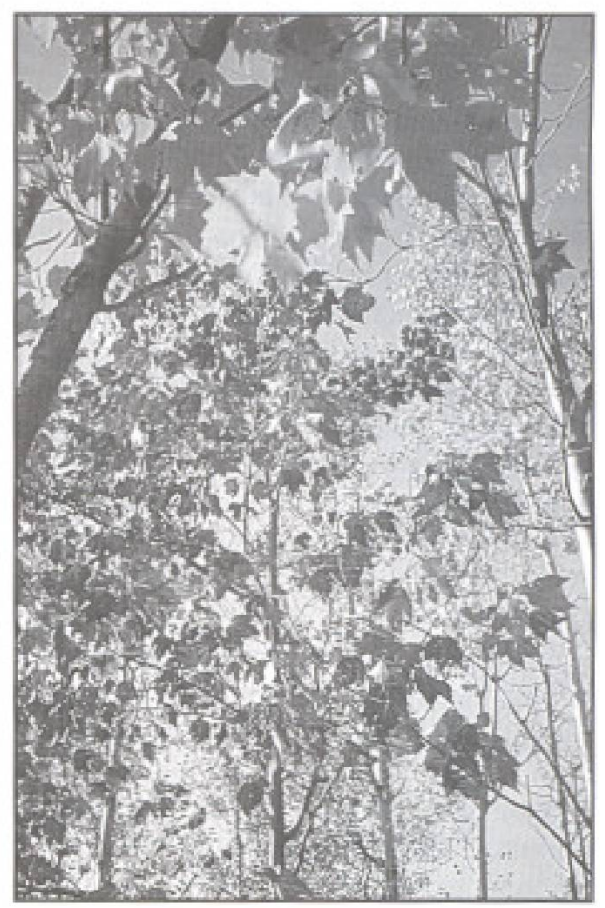


jardins historiques, 1'île de Montréal pourrait tirer profit de la couverture des publications de jardinage et attirer la clientèle qu'elle a identifiée dans ses études de marketing. Paris en est un bel exemple. La création et le réaménagement de jardins sont devenus un élément clé de l'attrait de cette ville culturellement généreuse. Plusieurs jardins et parcs, notamment le parc André-Citroën conçu par G. Clément, P. Berger, A. Provost, J-P Viguier et J-F Jodry, sont devenus une destination de choix pour les concepteurs et les architectes paysagistes, ainsi que des attractions majeures pour une clientèle spécialisée.

Le développement d'un jardin à l'arrière du Musée du Château Ramezay dans le Vieux Montréal est un indice que les jardins sont pris au sérieux par les urbanistes et les institutions. Ce jardin de 1,2 million $\$$ devrait ouvrit en juin 1999. Il ne s'agit pas d'un réaménagement étant donné que le Château Ramezay n'a jamais possédé de jardin comme tel, mais plutôt de l'amenagement $\mathrm{d}^{+}$un nouveau jardin inspiré du XVIII ${ }^{e}$ siècle. En prenant les mesures nécessaires pour réaménager et ouvrir les jardins du Vieux Séminaire sur la ne Notre-Dame dans le Vieux Montréal, la ville se doterait d'une destination touristique horticole de base. Le réaménagement depuis si longtemps nécessaire des jardins du Grand Séminaire sur la rue Sherbrooke pourrait être un complément naturel. Mais peut-être est-il déjà trop tard pour sauver un des plus importants jardins situés sur la propriété d'une communauté religieuse en Amérique du Nord.

Ëtonnamment, Montréal a cedé à d'autres villes la tenue d'événements horticoles. Fierre d'organiser des salons d'expositions et des événements horticoles pour les jardiniers du Québec, Montréal n'a pas tenu d'exposition horticole depuis les Floralies internationales en 1980. Elle a apparemment concédé les Floralies à la ville de Québec qui entend bien répéter le succès de son exposition de 1997 en 2002 , 2005 et 2008. Bien que la concession de l'exposition à Québec soit un êlément positif pour la diversification de l'industrie du tourisme, Montréal ne peut compter sur aucun évênement de jardinage d'envergure. Cela explique peut-être les projets mis de l'avant de tenir deux événements en l'an 2000 : une exposition florale printanière au Stade Olympique et une compétition internationale de a mosaïculture $x^{11}$.

\section{LA VILLE DE QUÉBEC : CAPITALE DU JARDIN HISTORIQUE EN AMÉRIQUE DU NORD ?}

Il appert que le développement du produit horticole au Québec doit compter sur les jardins à l'extérieur de Montréal. Le circuit des grands jardins le plus dense se trouve à Québec. Ầ proximité l'un de l'autre, on trouve le jardin Van den Hende, la villa Bagatelle, le domaine Cataraqui, le parc du Bois-de-Coulonge, la maison Henry Stuart et le jardin Jeanne d'Arc sur les Plaines d'Abraham. Chacun de ces jardins se distingue par son charme et son intérêt particuliers. Cependant, à l'exception du jardin Van den Hende de I'Université Laval, aucun de ces jardins n'est un jardin per se. Ce sont des propriétés ou des parcs historiques. L'histoire n'a pas toujours été tendre à leur égard. En 1961, le bâtiment principal du si magnifique domaine Spencerwood, maintenant connu comme le parc du Bois-de-Coulonge, a été détruit par un incendie. Mais dans l'ensemble, les jardins de la ville de Québec offrent une experience touristique solide, à un coutt raisonnable ou sans frais.

Un circuit touristique qui comprendrait les jardins historiques des Ursulines et les cimetières de Mount-Hermon et de St. Patrick enrichirait la sélection de jardins déjà abondante. On ne doit pas non plus oubliè le domaine Maizerets qui, avec son bâtiment historique, son étang et son nouvel arboretum aménagé au cout de 4 millions \$, vaut le détour. Le réaménagement du Jardin du Gouverneur, maintenant un parc public laissé à l'abandon près du Chatteau Frontenac, pourrait être un élément clé dans le développement des jardins comme un produit distinct de la ville de Québec. Le site, administré par Pares Canada, a fait l'objet d'études exhaustives, mais aucun travail d'amenagement paysager n'a encore été entrepris. Avec un peu d'investissement et un peu d'imagination, la ville de Québec pourrait facilement devenir la capitale du jardin historique en Amérique du Nord et un centre de tourisme horticole.

\section{HORS QUÉBEC}

Les jardins historiques les plus intêressants sont situés à l'extérieur des centres urbains. En moins de trente minutes de route de la

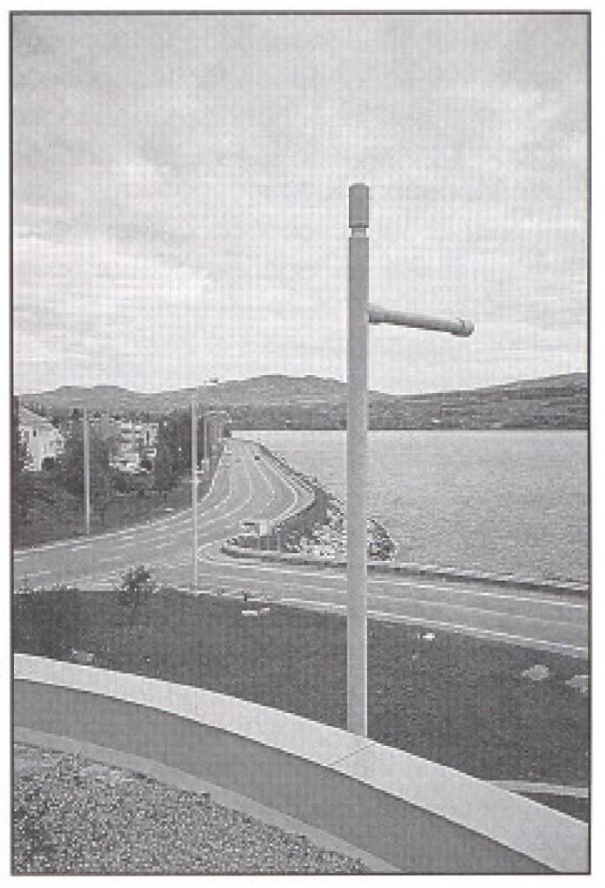

ville de Québec se trouve le domaine Joly= De Lotbinière. Les plans de développement de son nouveau propriétaire, la Fondation du domaine Joly-De Lotbinière, renforcent l'argument selon lequel les jardins sont un produit en voie de développement. En 1999. après la fin des travaux de restauration tant nécessaires du manoir seigneurial et de ses dépendances datant de 1851 , la Fondation projette de réaménager les jardins et d'en aménager d'autres. Ce projet donne suite en partie à l'évidence même que les jardins dotés de bâtiments historiques ont un nombre plus élevé de visiteurs que les bâtiments historiques entourés de jardins. Jouissant de l'ampleur des seigneuries et des domaines, l'intérêt pour de telles propriétés s'est rêvélé limité. Peu de ces sites attirent plus de 10000 visiteurs annuellement au Québec. Le domaine Joly est en voie de développer un produit distinct avec la mise en valeur des jardins et de la forêt, en plus de mettre sur pied un programme d'interprétation visant à expliquer la vie et les préoccupations des hommes et des femmes qui ont planté ces jardins. Defavorise par sa distance de l'autoroute 20 , le domaine Joly tire toutefois avantage de sa majestueuse propriété qui est l'une des rares seigneuries qui soit demeurée intacte au fïl des siecles dans la province. Compte tenu de son objectif de développer ses jardins à l'aide d'un apport financier adéquat, le domaine Joly se démarquera avec le temps comme l'un des jardins et l'une des propriétés historiques prééminents dans la province. 
Le domaine Mackenzie King se trouve de l'autre côté de Montréal. Cette propriété est déjà l'une des plus populaires au Québec. Le domaine de l'ex-premier ministre Mackenzie King n'est doté que d"un seul petit jardin fleuri, mais sa conception est inspirée des vastes jardins pittoresques. Caractérisée par sa collection de ruines idiosyncratiques choisies et disposées par son propriétaire, la propriété a un cachet unique. Populaire auprès des résidants des régions de l'Outaouais et d'Ottawa, le domaine est littéralement envahi de visiteurs durant la période des couleurs à l'automne. Relativement isolé des autres jardins du Québec, le domaine Mackenzie King pourrait profiter du développement des autres jardins de la région. Les circuits de jardins pourraient inclure alternativement d'autres jardins de la région, tels les jardins du Rideau Hall ou les aménagements paysagers de Calvert Vaux des édifices parlementaires à Ottawa.

Un autre partenaire encore plus prometteur est le Manoir Papineau à Montebello. Des travaux de réaménagement sont en cours par Parcs Canada, mais l'ouverture n'est pas prévue avant plusieurs années. Les jardins crés par Louis-Joseph Papineau sont parmi les plus attrayants sur le continent. La vie exotique de leur créateur, le parlementaire rebelle Louis-Joseph Papineau, la conception conforme aux préceptes des jardins pittoresques glanés par Papineau alors qu'il vivait en exil aux États-Unis et en France ainsi que la beauté naturelle de la propriété recèlent une myriade de possibilités. Le fait de partager une propriété avec le Château Montebello appartenant à la compagnie du Canadien Pacifique devrait faciliter le développement d'un marché de visiteurs. Les jardins Mackenzie King et Papineau pourraient facilement être intégrés à un circuit comprenant les communautés de Senneville et d'Hudson, l' Arboretum Morgan et les Jardins d'Henrictte à Rigaud, jardins privés mais accessibles au public.

A l'est de Montréal se trouve le jardin Daniel A. Séguin, le joyau des étudiants en horticulture de I' Institut de technologie agro-alimentaire de Saint-Hyacinthe. La ville de Sherbrooke prévoit une expansion majeure des jardins du domaine Howard dans le cadre de son bicentenaire en 2002. Les jardins et le bâtiment historiques seront dotés de plusieurs nouveaux jardins à être aménagés au cours des prochaines années à un coût approximatif de 2,3 millions $\$$.

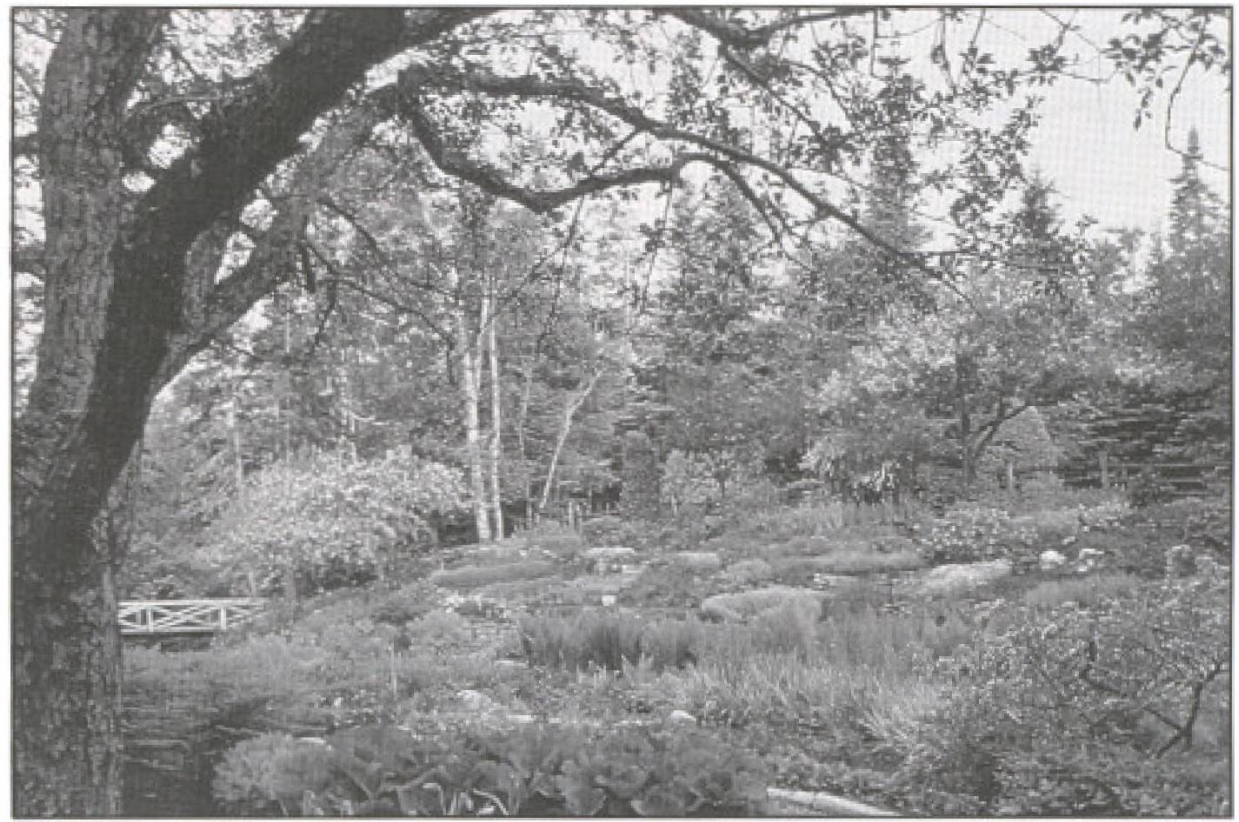

Le phénomène le plus notable dans le domaine des grands jardins du Québec est sans aucun doute les Jardins de Métis. Durant près de quarante ans, ces jardins ont affiché un nombre remarquable de visiteurs. Acquis par le gouvernement du Québec de la famille Reford en 1961, les jardins sont ouverts au public depuis 1962. Personne n'a été plus surpris que le directeur lui-même de constater que 16000 personnes avaient visité les jardins au cours du premier été d'exploitation. Depuis sa privatisation, le nombre moyen de visiteurs se chiffre à 95000 (de juin à la mioctobre), et à deux reprises le nombre a dépassé 100000 (1995 et 1998). Ce chiffre est remarquable étant donné que les Jardins de Métis sont relativement isolés d'un centre urbain $(350 \mathrm{~km}$ à l'est de la ville de Québec). Le chiffre magique de 100000 visiteurs est exceptionnel pour des jardins, même aux États-Unis et en Europe.

Une des raisons de ce succès est sans doute l'emplacement stratégique des jardins sur la route 132 au début du etour de la Gaspésies, qui demeure l'un des circuits routiers les plus populaires en Amérique du Nord. Métis est bien connu des touristes québécois depuis plus de trois décennies et sa réputation n'est plus à faire. Depuis leur privatisation en 1995, les Jardins de Métis ont su tirer avantage des publicités parues dans des magazines spécialisés en jardinage du Canada anglais. Mais, tout comme les autres jardins du Québec, ils attirent très peu de touristes de l'Ontario et du Canada anglais en général. À l'exception du domaine Mackenzie King, les jardins du Québec ont plus de visiteurs européens $(10$ à $15 \%)$ et américains $(5 \%)$ que de visiteurs canadiens anglais ( $4 \%$ ). Il en va de même pour la plupart des jardins du Québec qui continuent de s'appuyer sur les visiteurs québécois qui constituent de $60 \%$ (Jardin botanique de Montréal) à près de $100 \%$ de la clientèle de certains jardins ${ }^{12}$.

Les raisons de la popularité d'un jardin sont parfois difficiles à cerner. Bien que l'emplacement joue évidemment un rôle important, l'esprit des lieur est aussi important. Les jardins privés devenus publics sont souvent plus populaires que les jardins aménagés pour le public. Ces derniers ont un caractère institutionnel, mais sont dépourvus de l"intimité des jardins privés et de la dimension recherchée par les visiteurs. Les jardins botaniques et leurs émules sont souvent trop ouvertement didactiques pour les jardiniers qui recherchent de l'information indirectement plutôt que directement. Bien équilibrer les intérêts des professionnels de l'horticulture et ceux des jardiniers est difficile à réussir. Le succès de certains jardins, comme les fameux Butchart Gardens près de Victoria en Colombie-Britannique, repose sur la combinaison de l'intimité d'un jardin privé et des plantations flamboyantes d'un jardin public. Bien que les jardins ne soient pas universellement acclamés par les puristes, ils sont remarquablement efficaces pour attirer le grand public avec plus d'un million de visiteurs annuellement. Aucun jardin du Québec situé hors d'un centre urbain ne pourra jamais connaître 
un tel achalandage. Mais les Jardins de Métis sont un exemple que la combinaison de l'emplacement, du marketing et de la personnalité des créateurs peut plaire à un vaste public.

Les Jardins de Métis sont à mettre sur pied des projets ambitieux dans le but de devenir une véritable destination touristique. Ces mesures résultent d'une longue démarche de réflexion et de planification. Pour se protéger contre les fluctuations du nombre de touristes en Gaspésie, on mise sur le développement de programmes éducatifs fondés sur les jardins. Une série d'activités sont prévues pour faire des jardins un centre d'ateliers de jardinage, de stages, de cours d ${ }^{*}$ été, de conférences et de symposiums. On prépare également un festival annuel des jardins contemporains prévu pour l'an 2000 et les années a venir. On dotera l'entrée du site d'un nouveau complexe pour les visiteurs. On ajoutera même des jardins pour des activités pédagogiques et un jardin de plantes rares et menacées d'extinction du Québec et du bassin du Saint-Laurent. Ces projets évalués a 3,5 millions $\$$ se concrétiseront au cours des cinq prochaines années. Le gain net sera une augmentation du nombre de visiteurs évaluée à 125000 et une stabilisation de ce nombre.

Le succès des Jardins de Métis en a encouragé d'autres à rêver au développement des jardins dans leur propre communauté. Le plus ambitieux de ces projets est sans nul doute celui des Grands Jardins de Normandin. C'est monsieur Gérald Bélanger qui a d'abord eu l'idée de recréer à Normandin les célèbres jardins de Villandry de la Vallée de la Loire en France. Les jardins ont ouvert en 1996. Avec leurs 55 hectares, les couts de construction ont excédé 3 millions $\$$. Au cours de leur première année d'exploitation, les jardins ont accueilli 30000 visiteurs. Par la suite, le nombre de visiteurs a augmenté, mais pas nécessairement au rythme prévu et espéré.

A Cabano, l'organisme qui exploite le site historique Fort Ingall, un fort recréé sur les rives du Lac Témiscouatá dans 1'Est du Québec, a eu l'idée de construire une roseraie pour augmenter le nombre de visiteurs. La roseraie a été construite au coût de 130000 \$. Les jardins ont affiché un nombre respectable de 9000 visiteurs en 1998.
Un autre jardin du Québec pourrait facilement devenir une attraction touristique d'envergure internationale. Les jardins de Monsieur Francis H. Cabot à La Malbaie sont reconnus comme étant parmi les plus beaux et grands jardins aménagés depuis la Seconde Guerte mondiale ${ }^{13}$. Déjà une Mecque pour les passionnés de jardins, ils sont ouverts sur une base limitée à plusieurs reprises chaque êté. M. Cabot a récemment annoncé que les jardins seront complètement fermés au public en l'an 2000 , ce qui n'est pas surprenant en soi. car les jardins font partie d"un domaine d'été privé, et ils n'ont pas été conçus ou aménagés pour accommoder un grand nombre de visiteurs. Le phénomene des Quatre Vents a eu son lot de retombees. D'autres jardiniers ont commencé à ouvrir leurs jardins privés au grand public sur une base payante. Bien qu'aucun jardin ne puisse Égaler l'ćchelle et l'excellence des Quatre Vents, le phénomène vaut la peine d'être souligné. En mettant sur pied un système à l'échelle provinciale, comme il en existe en France et dans d'autres pays, les jardins privés pourraient être ouverts au grand public le(s) même(s) jour(s) partout dans la province. Par exemple, les deuxièmes samedis de juin, de juillet et d'août pourraient devenir les jours consacrés aux jardins au Québec. Il appert que c'est seulement de cette façon que les jardins privés pourraient devenir partie integrante du tourisme horticole.

Le phénomène de l'aménagement de jardin n'est pas unique au Québec. Durant la dernière décennie, deux jardins ont étét construits au Nouveau-Brunswick, un à la frontière du Québec et l'autre à St. Andrews. Le Jardin botanique du Nouveau-Brunswick situé à Saint-Jacques a été construit vers la fin des années 1980. Depuis lors, il a connu un certain succès auprès de la population, mais les coûts élevés liés à l'exploitation des jardins ont résulté en une multitude de changements au niveau de la direction. Depuis peu, les jardins sont administrés par l'Université de Moncton.

A St. Andrews, Lucinda et John Flemer ont offert leur support pour le développement; la conception et l'aménagement d'un jardin sur la propriété familiale de madame Flemer. La propriétế, une des nombreuses à St. Andrews qui ont été développées par des familles montréalaises au début du siècle, regroupe un bâtiment et des plantations historiques. Les concepteurs ont créc une série de jardins en respectant le cadre historique, 8 "appropriant les tendances contemporaines de la conception de jardins dans un environnement traditionnel. Construits au coût de 1,8 million $\$$, les jardins ont reçu 22000 visiteurs lors de leur premier été d'exploitation en 1998.

\section{L'ASSOCIATION DES JARDINS DU QUÉBEC}

L'organisme responsable de la promotion des jardins de la $*$ belle province $*$ est l'Association des jardins du Québec.

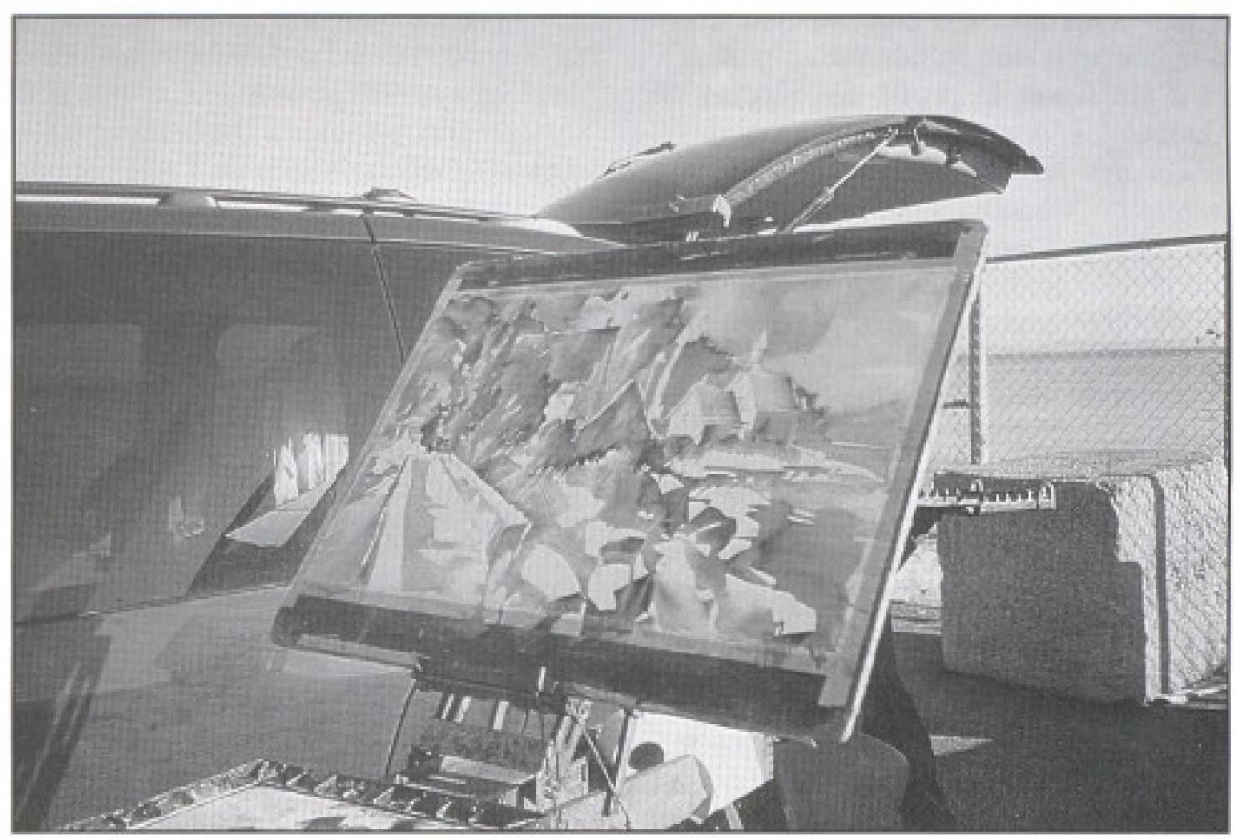




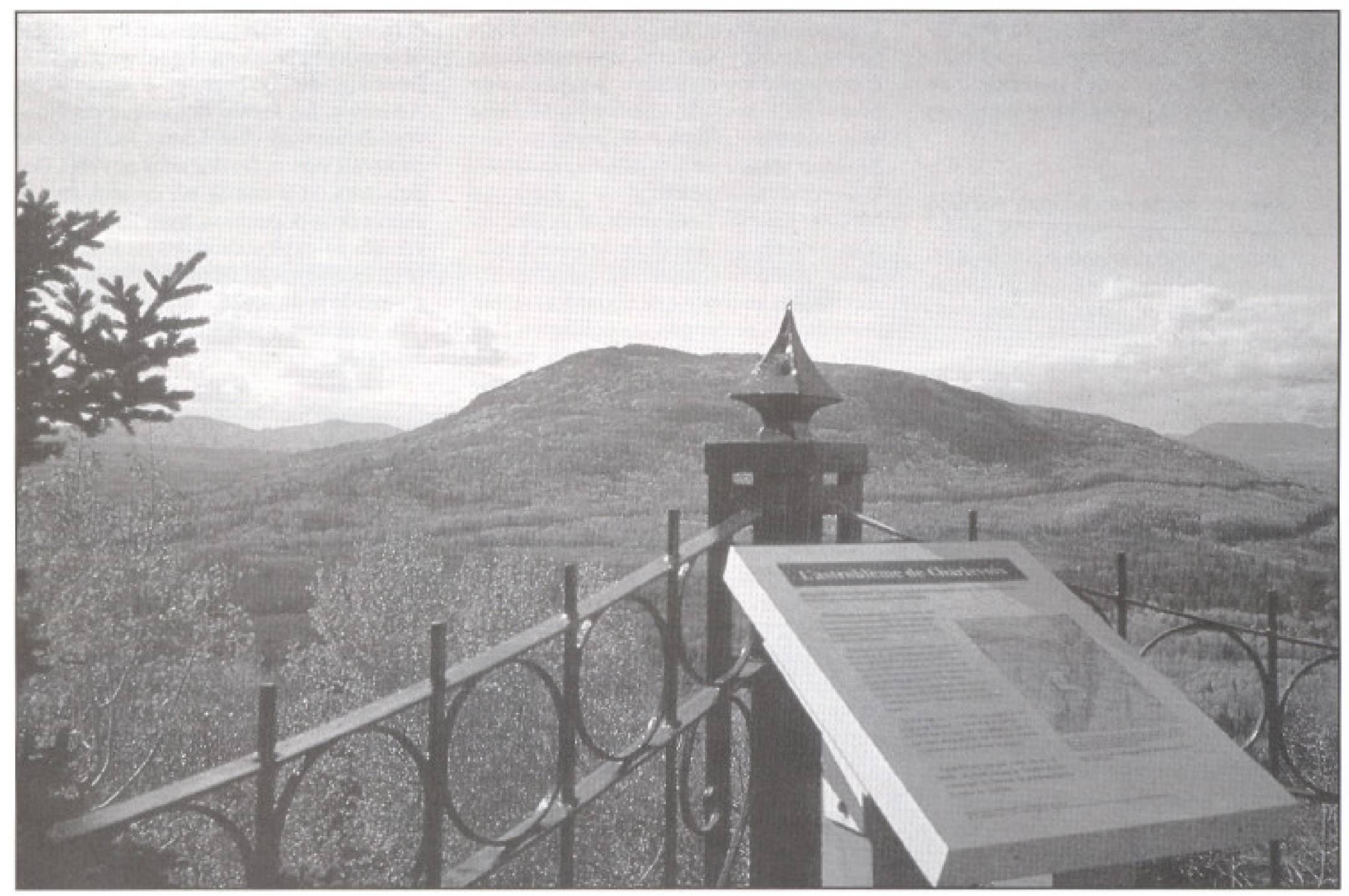

Actuellement, vingt jardins sont membres de l'Association qui a vu le jour en 1990 pour donner suite à une initiative du Parc des Champs-de-bataille de la ville de Québec. Les sept grands jardins des Plaines d'Abraham ont été regroupés dans le but d'encourager une collaboration mutuelle et d'améliorer le profil des jardins du Québec ${ }^{14}$. L'Association était alors réservée à ces jardins seulement. Dans les années qui ont suivi, l'Association a élargi ses critères d'adhésion et son nombre de membres a graduellement augmenté pour inclure la plupart des jardins de la province. Les jardins privés ont généralement été exclus de I'Association jusqu'au jour où ils ont pu offrir aux touristes un attrait et un entretien satisfaisants, ainsi qu'une gamme complète de services (toilettes, stationnement, tours guidés, heures d'ouvertures régulières, etc.).

L'Association a existé comme une association collective de marketing, du moins durant la majeure partie de son existence. Les frais d'adhesion annuels étaient tout d'abord utilisés pour la publication et la distribution d'une brochure annuelle et pour faire parâttre de la publicité dans des magazines sélectionnés. Plus récemment, $1^{9}$ Association a élargi son mandat en offrant sa collaboration à la production de deux livres traitant des jardins au Québec et. de concert avec Les Editions Continuité. de guides sur les jardins au Canada. Elle s'est de plus associée à Tourisme Québec et au MAPAQ (ministère de l'Agriculture, des Pecheries et de l'Alimentation du Québec) ; elle offre également son support au Musée du Château Ramezay dans la préparation d'une exposition sur le jardinage au Québec dont le lancement se fera dans le Vieux Montréal en juin 1999 conjointement avec l'ouverture du anouveaus jardin du Musée, jardin inspiré du XVIII siecle.

Il existe peu d'associations similaires à l'Association des jardins du Québec en Amérique du Nord. Ce n'est que récemment qu'une association ayant le même mandat a vu le jour en Ontario. Ni la Colombie-Britannique ni les provinces de l'Atlantique n'ont d'association pour assurer le regroupement des jardins dans leur province respective. Aux Etats-Unis, la plupart des jardins sont membres de l'American Association of Botanical Gardens and Arboreta, mais les services offerts par cette association sont pour la plupart destinés aux jardins botaniques. Le Garden Conservancy, fondé en 1989 par Francis H. Cabot (des Quatre Vents à La Malbaie), a mis de l'avant des mesures pour sauver plusieurs jardins négligés. Cette institution organise des visites de jardins, mais ses efforts visent principalement la préservation des jardins historiques plutôt que leur promotion. On trouve des associations de jardins dans plusieurs régions, comme les jardins Brandywine Valley au Delaware conçus par les membres de la famille DuPont qui, a eux seuls, font de la région une destination horticole de choix. Il existe beaucoup moins d'associations dans les états américains qu'on ne pourrait l'imaginer. L'interêt des jardins individuels (et leur dotation), comme le Biltmore Estate ou les Longwood Gardens, est peut-être tel que ces jardins ne nécessitent pas le support d'un regroupe- 
ment pour en faire la promotion. Les Butchart Gardens à Victoria sont les seuls jardins au Canada qui jouissent d'une réputation et d'un nombre de visiteurs comparables.

\section{DES JARDINS EN PLEINE CROISSANCE}

Pour permettre aux jardins du Québec de devenir un produit touristique cohésif en soi, de plus grands efforts doivent être entrepris pour développer le tourisme horticole et coordonner les efforts de vente. La stratégie récemment mise en ceuvre par Tourisme Québec avec le concours de l'Association des jardins du Québec (brochures, marketing direct, participation à des salons professionnels) augmentera avec le temps la visibilité des jardins du Québec, A ce jour, I+ OCTGM roffice des Congrès et du Tourisme du Grand Montréal) a entrepris ses propres efforts de vente conjointement avec le Jardin botanique de Montréal.

Parallelement, on devra proposer aux organisateurs de voyage des itinéraires touristiques horticoles et culturels intéressants qui se vendent bien. Les jardins devront de leur côté s'ajuster à la situation. En plus de publier des brochures et du matériel promotionnel en français et en anglais, ils devront s'assurer que leurs jardins répondent aux notmes de qualité auxquelles stattend le touriste horticole, plus averti et exigeant que le touriste moyen. Les jardins devront également pouvoir offrir des services à la clientèle dans la langue de leur choix. A l'heure actuelle, les jardins à l'extérieur de Montréal et de la région de l'Outaouais ne sont pas adéquatement équipes pour offrir des circuits et des services bilingues.

Une des raisons qui expliqueraient l'approche plutôt désordonnée du développement des jardins au Québec est l'absence d'un partenariat avec un ministêre ou une agence spécifique qui aurait pour mandat de superviser leur développement. La variété de types de propriétés est en cause à cet égard. Certains jardins ne sont pas la propriété de sociétés à but lucratif ou de fondations, alors que d'autres sont administrés par des universités ou des collèges, ou sont des propriétés privées. Plusieurs sont administrés par des agences fédérales ou provinciales. Dans tous les cas, le taux de financement est variable.

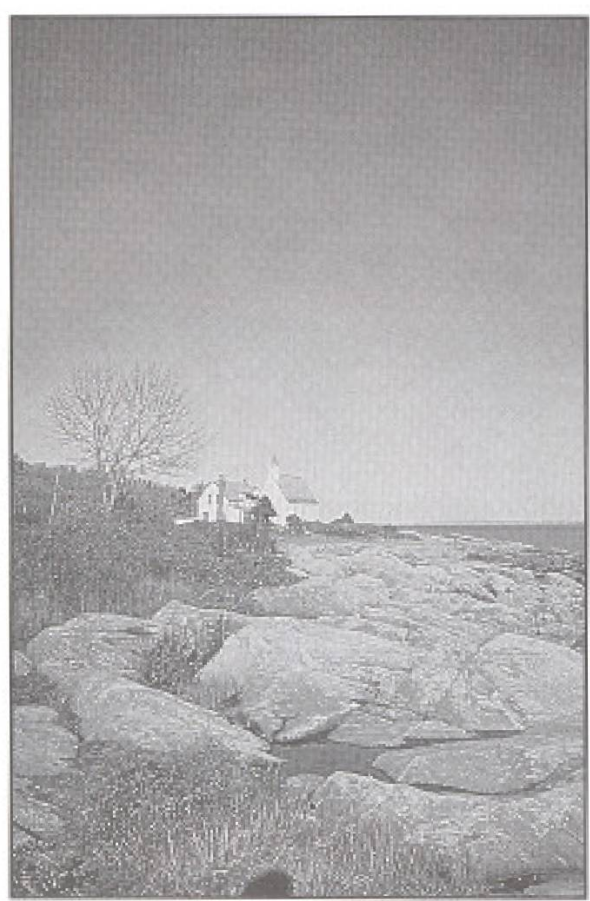

Certains jardins sont accrédites par le ministêre de la Culture et des Communications (MCC) comme sites d'interprétation (Seigneurie des Aulnaies) et d'autres comme musées (Domaine Cataraqui). I1s reçoivent ainsi une subvention annuelle pour leur exploitation. La plupart des jardins, toutefois, n'en reçoivent pas. Quant à l'Association des jardins du Québec, elle opère avec un budget limité et sans effectifs. Elle reçoil une modeste contribution annuelle de $1000 \$ \mathrm{du} \mathrm{mi-}$ nistere de la Culture et des Communications. Autrement, elle s'autofinance avec les frais d'adhésion de ses membres.

Les jardins du Québec pourraient bénéfi= cier du parrainage avec un ou plus d'un ministère gouvernemental, Au Québec, les deux choix les plus évidents sont le MAPAQ et le MCC. Alors que le MAPAQ est un joueur clé dans le développement de l'industrie horticole du Québec, il s'est limité jusqu'à ce jour à un rôle négligeable dans le développement des jardins du Québec. Contraints dans leur mandat d'aider l'industric, le MAPAQ et le MCC ont réduit leur champ d'application à soutenir l'industrie horticole. Le rôle des jardins du Québec comme moteur de visibilité des créations horticoles des jardiniers québécois a été négligé, tant par l'industrie que par le ministère. Très récemment, le MAPAQ et l'Association des jardins du Québec ont conclu une entente pour organiser une partie des activités du concours annuel Fleurir le Québec auquel participeront les jardins membres. Les deux parties examinent de plus dans quelles mesures ils pourraient élargir leur collaboration.

Patrimoine Canada octroie rarement des subventions aux jardins. Bien que la définition fédérale de amuséesw inclut les jardins, les rendant ainsi admissibles à des subventions pour des projets spécifiques, les critêres d'admissibilité à de telles subventions excluent souvent les jardins. Agriculture Canada a contribué aux Floralies 1997 et aux Floralies 2002, mais ce ministère limite ses contributions à soutenir l'industrie horticole ou à développer de tels événements spéciaux ${ }^{15}$.

Le financement des jardins du Québec provient largement des budgets des agences de développement régional, soit les Conseils régionaux de développement (CRD) et leur homologue fédéral, Développement économique Canada. Bien que cela ne soit pas en soi inapproprié, il appert que les agences dotées de la plus grande expertise (ministère de la Culture et des Communications, et Pares Canada) sont parfois exclues de jouer un röle actif dans le développement des jardins du Québec.

\section{LES JARDINS HISTORIQUES DU QUÉBEC}

La croyance générale veut que l'intérêt pour le jardinage au Québec remonte aux Floralies tenues à Montrếal en 1980, mais la province possède un héritage de jardinage beaucoup plus riche. Le jardin du Vieux Séminaire dans le Vieux Montréal est consideré comme le jardin le plus âgé sur le continent nord-américain. Même si les jardins aménagés sur les propriétés des communautés religieuses de Montréal et de la ville de Québec sont en gếnéral dans un état lamentable et ne sont pas ouverts au public, ils n'en demeurent pas moins le gage d'un intérêt dans le jardinage qui date des premières années de la colonisation.

L'histoire du jardinage n' a pas tenu compte des jardins du Québec ni de ceux du Canada en géneral. Dans le Oxford Companion to Gardens, on note dans l'introduction sur le Canada: \& If one defines garden art in terms of the great classical and romantic garden movements supported by wealthy private patrons, then Canada cannot be said to have developed a great 
garden tradition $^{16} \mathrm{~m}$. Il va sans dire que le Québec ne peut rếclamer un héritage aussi fertile que celui des régions de l'Europe et de l'Est des États-Unis, mais la province possède un important patrimoine de jardins, bien qu'encore méconnu et peu documenté.

La conservation, la revalorisation, le réaménagement et la reconstruction des jardins historiques sont devenus des préoccupations majeures sur le plan international depuis les dix demières années. Le mouvement de protection des jardins a pris naissance en Europe, où une série de conférences dans les annees 1970 sur la conservation et le réaménagement des jardins historiques tenues par l'ICOMOS (International Council on Monuments and Sites), subventionné par l'UNESCO, ont conclu à l'établissement d'une charte sur la préservation des jardins historiques, maintenant connue comme la Charte de Florence.

Alors que ces débats étaient confinés â une communaute relativement insulaire, ils ont néanmoins été une source précieuse d'information sur la préservation du patrimoine à l'intention des intervenants des gouvernements. Les approches identifiees dans la Charte de Florence ont été plus au moins. mises en application au Québec. Le groupe d'experts associés a Parces Canada a utilise un large eventail de techniques en évaluant, entre autres, l'évolution du Jardin du Gouverneur à Québec. Dans le même ordre d"idées, le Manoir Louis-JosephPapincau à Montebello a fait l'objet d'ctudes exhaustives ${ }^{17}$. Le réaménagement des deux jardins demeure toutefois au stade de planification seulement.

Au Québec, le ministère tout d'abord intéressé au dossier du réaménagement des jardins du Québec a été le ministère de la Culture et des Communications, Ce ministère a áté impliqué dans l'évaluation de l'état des jardins historiques du Québec. Une grande partie de leur travail áté faite dans les environs de la ville de Québec. où les jardins historiques du chemin SaintLouis étaient autrefois abondants et magnifiques. Le ministère a financé en partie ou en totalité les études du domaine Cataraqui, du pare du Bois-de-Coulonge. de la villa Bagatelle et de la maison Henry Stuart. Dans la plupart des cas, ces études ont résulté en un réaménagement d'au moins une partie de ces jardins et a $1^{*}$ adoption d'une approche globale de leur mise en valeur.

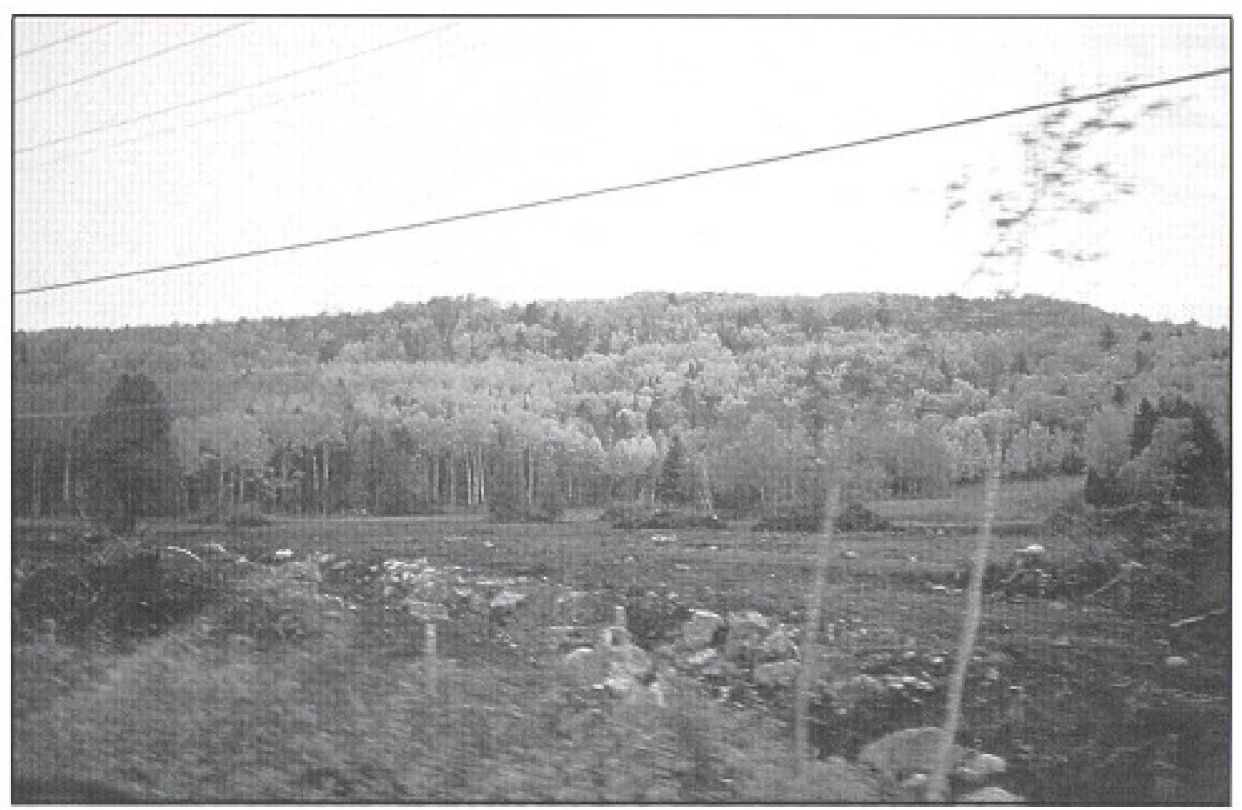

Ailleurs en province, d'autres ministères ont pris les devants. A Métis, le ministère du Loisir, de la Chasse et de la Péche a mandaté Carole-Fernet et Associés à entreprendre une êtude sur les Jardins de Métis en 1992. Le plan de développement soumis par la firme souligne l'importance de développer les jardins dans l'esprit de leur créateur, madame Elsie Reford, mais les jardins ont b́té privatisés avant qu'une seule des recommandations ne soit mise en application. Plus récemment, le même ministère a mandaté Jacques Des Rocher's pour faire l'évaluation du jardin Pease situe aux frontières du pare du Mont SaintBruno. Son étude approfondie de l'histoire des jardins et de ses propriétaires a été à la base des plans du site actuellement en développement ${ }^{\text {ts. }}$. Le jardin doit être réaménagé dans les années à venir.

De façon générale, le nouvement pour le réaménagement des jardins historiques du Québec a insufflé le réflexe d'entreprendre une étude sur l'histoire du jardin avant de procéder à son réaménagement, une étape qui constitue une des exigences pour que les jardins soient considérés au classement. Jusqu'à récemment, ni les sites naturels ni les jardins n'ont été considérés admissibles au classement en vertu de La loi sum les biens culturels (L.R.Q., chapitre B-4) ${ }^{19}$. Il est maintenant largement reconnu que les sites plutôt que les bâtiments devraient recevoir le classement. Une des conditions du classement est que le site établisse un plan directeur, un document ayant pour but de guider le développement du site dans son ensemble. Les demandes de classement pour des sites tels le Jardin botanique de Montréal, le Manoir Fraser (Rivière-duLoup) et le domaine Joly-De Lotbinière sont présentement devant la Commission des biens culturels du Québec et ils donneront vraisemblablement suite à de tels criteres. Les jardins n ont parfois pas les ressources nécessaires pour entreprendre de telles études et, plus souvent qu'autrement, pour assurer leur mise en application. Le classement en soi ne garantit pas le réaménagement d'un jardin ni même son entretien. Certains jardins, comme ceux du Vieux Seminaire et du Grand Sếminaire de Montréal, ont fait l'objet d'ếtudes exhaustives et ont reçu un classement, mais ils doivent toujour's être réaménagés et sont entretenus de façon minimale.

A ce jour, les groupes intéressés à la préservation des paysages du Québec, comme les Etats généraux du paysage québécois, sont demeurés, de façon surprenante, muets au sujet des jardins du Québec. Puisque les parcs et les réserves forestières sont déjà des sites protégés, les jardins sont peut-être considérés comme une moindre priorite. Mais les jardins, particulièrement ceux qui appartiennent à de grands domaines, peuvent procurer d'importantes leçons sur la préservation du paysage. L'événement tenu récemment dans Charlevoix, auquel participait le Glynwood Center (voir l"article de Judith Labelle), prenait en consideration le rôle du jardin dans le paysage. Ailleurs, les Jardins de Métis travaillent conjointement avee la Chaire en paysage et environnement de l'Université de Montréal pour utiliser les jardins 
et les sites comme un observatoire du paysage, ce qui permettra de tracer l'évolution du paysage au cours du siècle dernier. Une telle approche pourra confirmer le rôle du jardin dans le façonnement de l'environnement local. Il permettra de plus de faire état de l'insensibilité générale dếmontrée par d'importants joueurs régionaux, tels Hydro-Québec et le ministère des Transports, à l'égard du paysage local dans les années passées.

Un élément important dans la promotion des jardins du Québec est de s'assurer que les jardins soient d'une qualité exceptionnelle, en termes de conception, de plantations et d'entretien. Il est tout aussi primordial que l'histoire du jardinage au Québec soit mieux connue. De récentes publications, notamment Promenades dans les jardins anciens du Québec (Boréal, 1996), ont permis de faire la lumiere sur l'histoire des jardins. De façon similaire, un livre écrit et préparé par Alexander Reford à être publié en mai par Les Publications du Québec, Les jardins antiques du Québec, illustrera plus de 100 jardins du Québec photographiés depuis la moitie du XIX siecle. Ces initiatives et bien d'autres aideront les jardins du Québec à se faire mieux connaître et apprécier partout au Québec et à l'extérieur de la province.

\section{CONCLUSION}

Par chaque mesure mise de l'avant, les jardins du Québec sont en voie de développement. Variant d'une région à l'autre. d'un site à l'autre, les jardins sont devenus de façon indiscutable des joueurs clés pour le tourisme au Québec. Les efforts entrepris par l'Association des jardins du Québec, et plus récemment par Tourisme Québec, favoriseront la promotion des jardins, particulièrement à l'extérieur de la province. Les régions qui ont récemment aménagé des jardins devtont faire preuve de créativité pour se doter d'outils qui favoriseront leur développenent. Et les régions qui possèdent des jardins populaires devraient reconnaître leur importance dans le développement régional. Il serait judicieux pour les gouvernements qui ont beaucoup investi dans le développement de nouveaux produits culturels de porter une plus grande attention aux jardins, dont le profil de la clientèle et les plans de développement concordent en tous points avec les tendances démographiques et les habitudes changeantes des touristes. Les touristes ont déjà reconnu les multiples attraits des jardins du Québec et continueront de le faire, de plus en plus nombreux, dans les années à venir:

Alexander Reford est directeur de Les Jardins de Métis et president de l'Association des jardins du Québec, Il travaille depuis plusieurs annees dans le domaine de la mise en valeur des jardins historiques.

Un comitó de lecture a lu et accepté ce texte

\section{NOTES}

1 Agriculture et Agro-alimentaire Canada, Sitwation et tendances 1997-98, Industrie canadienne de la floriculture, des pépinières et des arbves de Noel, avril 1998 ; Aperçu de l'industrie horticole, Situation et lendances canadiennes - 1997-1998, 10 avril 1998.

2 Arboretum Morgan (Senneville), Centre de la nature de Laval, Jardin botanique de Montréal, Jardin Dąniel A. Séguin (SaintHyacinthe), Parc Marie-Victorin (Kingsey Falls), Parc du Bois-de-Coulonge (Québec), Domaine Joly-De Lotbiniẻre (Sainte-Croixde-Lotbinière), Domaine Cataraqui (Québec). Domaine Maizerets (Québec), Jardin Jeanne D'Are (Québec), Jardin Roger Van den Hende (Université Laval), Maison Henry Stuart (Québec), Domaine Howard (Sherbrooke). Jardins à fleur d'eau (Stanbridge East), Domaine Mackenzie King (Cheslea-Aylmer), Grands Jardins de Normandin (Normandin), Roseraic de Témiscouata (Cabano), Seigneurie des Aulnaies (Saint-Roch-des-Aulnaies), Jardin des Fleurs (Saint-Pâcome), Jardins de Metis (Grand-Métis).

3 Les critères d'adhésion pour devenir membte de l'Association comprennent : etre ouvert au public durant l'élé, posséder l'infrastructure nécessaire pour recevoir des visiteurs, y compris les groupes, devenir membre pour une période de trois ans, faire la promotion des jardins du Québec, permettre que les jardins soient évalués par un comité de pairs pour garantir leur intérett (horticole, arboricole et architectural), la qualité de l'entretien des jardins et des bâtiments, et la qualité des services offerts aux touristes.

4 Association des jardins du Québec, novembre 1998.

5 Les visites par les membres de l'Arboretum Morgan ne sont pas incluses dans ces données qui représentent les visiteurs payants seulement.

6 Nombre de visiteurs pour une période de 12 mois.
7 Le nombre exact d'entrées n'est pas disponible pour les jardins ouverts au public gratuitement. Par exemple : le parc du Bois-deCoulonge, les jardins. Jeanne d'Arc (Québec) et le Centre de la nature (Laval). Les jardins du domaine Howard estiment le nombre de leurs visiteurs entre 8000 et 10000 .

8 Office des congrès et du tourisme du Grand Montréal. Une approche créneau : "Les Gardener's Clubs w, 27 octobre 1997.

9 Ibid., p. 3.

10 \& Sweet Intervention *, Landscape Architecfure, avril 1998 , p. 20-21

11 "Le Québec en mosaïcultures \%, LActualite, $23,17, \mathrm{p} .14$.

12 Association des jardins du Québec, novembre 1998.

13 La littérature sur Les Quatre Vents est abondante. Liste partielle : Cominuite (été 1987); Quatre-temps (automne 1988); Gardens Whastrated (novembre 1996); Jardins Passion (automne 1997); Horticulture (avril 1998); House and Ganden (novembre 1998).

14 Jardin botanique de Montréal, Jardins de Métis, jardin Van den Hende, parc MarieVictorin, parc du Bois-de-Coulonge et domaine Maizerets.

15 La Société des Floralies internationales de Québec, Reminiscences Quebec en Jleurs 97 , Québec en fleurs 2002. Le Soleil, 28 septenbre 1998.

16 Hough, Michael (1991), Canada, The Otford Companion to Garden, Oxford, p. 89.

17 Prud'Homme, Chantal, Etude sur les jardins et le potager, mars 1995 : Zvonar, John E. Elude préliminaire de laménagement paysager, fevrier 1995 ; Zvonar, John E et Luc Berard, Etwde préliminaire de l'amenagement paysager, novembre 1995 ; Chassé, Béatrice, Le manoir Papineau à Montebello ; une survivance de la fédalité sur les bords de l'Outaouais, novembre 1979 ; Sylvico, Ine., Inventaire biophysique du lieu historique national du Manoir Papineau - Rapport final, septembre 1994.

18 Des Rochers, Jacques, Etude historique et analyse patrimoniale en vue d'éablir la pertinence du rélablissement d'un jardin au Mont Saint-Brano, avril 1994.

19 Ministère des Affaires culturelles, Le statur du bien culurel et ses effets, mai 1986 ; Gelly, Alain, Louise Brunelle-Lavoie et Corneliu Kirjan, La passion du patrimoine, La Commission des biens culturels du Québec $1922-$ 1994, Montréal, 1995, p. 224-227. 